\title{
Correction to: Gross hematuria after SARS-CoV-2 vaccination: questionnaire survey in Japan
}

\author{
Keiichi Matsuzaki $i^{1,2} \cdot$ Ryousuke Aoki $^{1,3} \cdot$ Yoshihito Nihei $^{1,3} \cdot$ Hitoshi Suzuki $^{1,3} \cdot$ Masao Kihara $^{1,3} \cdot$ Takashi Yokoo $^{1,4}$. \\ Naoki Kashihara ${ }^{1,5} \cdot$ Ichiei Narita $^{1,6} \cdot$ Yusuke Suzuki ${ }^{1,3,7}$ (1)
}

Published online: 29 November 2021

(C) Japanese Society of Nephrology 2021

\section{Correction to: Clinical and Experimental Nephrology https://doi.org/10.1007/s10157-021-02157-x}

In the original publication, the author has found 2 errors as below:

1. Methods: The text "Then, between June 9 and June 19, 2021, a second survey was emailed to the members who reported cases of gross hematuria" should read as "Then, between July 9 and July 19, 2021, a second survey was emailed to the members who reported cases of gross hematuria".

2. Figure 1 (under second survey): The duration of the months has been inadvertently appeared as June 2 to June 20, 2021, the corrected duration is July 9 and July 19, 2021. The corrected Fig. 1 given below.

The original article can be found online at https://doi.org/10.1007/ s10157-021-02157-x.

Yusuke Suzuki

yusuke@juntendo.ac.jp

1 Joint Research Team From Japanese Society of Nephrology and the Progressive Renal Diseases Research, Research on Intractable Disease, from the Ministry of Health, Labour and Welfare of Japan, Special Study Group for IgA Nephropathy, Tokyo, Japan

2 Kyoto University Health Service, Kyoto, Japan

3 Department of Nephrology, Juntendo University Faculty of Medicine, Hongo 2-1-1, Bunkyo-ku, Tokyo 113-8421, Japan
4 Division of Kidney and Hypertension, Department of Internal Medicine, Jikei University School of Medicine, Tokyo, Japan

5 Department of Nephrology and Hypertension, Kawsaki Medical School, Kurashiki, Japan

6 Division of Clinical Nephrology and Rheumatology, Niigata University Graduate School of Medical and Dental Sciences, Niigata, Japan

7 Division of Nephrology, Department of Internal Medicine, Faculty of Medicine, Juntendo University, 2-1-1 Hongo, Bunkyo-ku, Tokyo 113-8421, Japan 
Fig. 1 Outline of this study

First survey

June 2 to June 20, 2021

Councilor members of the Japanese Society of Nephrology (581 members in 382 facilities)

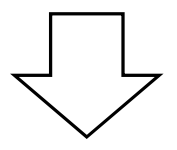

72 members reported 27 cases

(response rate: $18.8 \%$ of facilities)

Second survey

July 9 and July 19, 2021

Members who reported cases of gross hematuria

(27 members in 25 facilities)

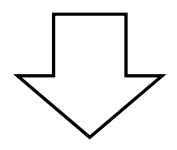

25 members returned the

information of cases

(response rate: $92.6 \%$ of cases)

Publisher's Note Springer Nature remains neutral with regard to jurisdictional claims in published maps and institutional affiliations. 